\title{
Optics in Polymer Science and Technology
}

\author{
Maksymilian Pluta, FELLOW SPIE \\ Institute of Applied Optics \\ 18 Kamionkowska Street \\ 03-805 Warsaw, Poland \\ E-mail: iosto@frodo.nask.org.pl
}

\author{
Andrzej Wasiak \\ Polish Academy of Sciences \\ Institute of Fundamental Technological Research \\ 21 Świętokrzyska Street \\ 00-049 Warsaw, Poland
}

\begin{abstract}
Our initial contacts focused on more than 20 potential authors all over the world, and from those we obtained about 15 proposals. Several of them, however, dealt with polymer science and technology in optics rather than optics in polymer science and technology, the title of this special section, and thus they were rejected. In general, the topic "polymers in optics" has become very popular during the last decade, and constructing a special section on that topic would be much easier than on its reverse, i.e., "optics in polymers."
\end{abstract}

Moreover, an international conference on refractometry held May 16-20, 1994, in Warsaw, Poland, preceded the contacts previously mentioned. The conference was chaired by one of us (Pluta), who stated that a number of conference papers fitted perfectly into the topic of our special section, but only one author from France, G. Vitrant, delivered two versions of his paper. The shorter one ${ }^{1}$ was published in SPIE/PL Proceedings, Volume 2208 and the full version is included in this special section. By contrast, this was not the case for three other papers from Hungary, ${ }^{2}$ Spain, ${ }^{3}$ and Russia ${ }^{4}$ on, respectively, reflectometry of multilayers of nonlinear optical polymers, measurements of refractive indices and thickness of polymer layers and foils by using a spectrophotometric interference technique with variable tilt of the sample under study, and determination of refraction and absorption indices of thin polymer films via frustrated total internal reflection (FTIR) spectroscopy. We regret that these excellent papers are outside the special section topic; the SPIE/PL volume mentioned before took priority over this section. Such a situation is selfevident because one of us (Pluta) was responsible for that volume and his interest was in preparing not only a good special section on optics in polymers but also an excellent SPIE/PL book on refractometry to commemorate the seventh anniversary of the Polish Chapter of SPIE in association with SPIE's 40th anniversary.

It is also worth noting that a valuable paper on reflection variant of nonlinear polarization spectroscopy was received from Belarus. ${ }^{5}$ The proposed method appears to be suitable for the study of anisotropic polymeric systems, but because of its high theoretical capacity the paper has been moved to Optica Applicata, a scientific journal published by the Institute of Physics at the Technical University of Wrockaw, Poland, since 1970 (the 25th anniversary of this journal is just coincident with the jubilees mentioned before.)

On the other hand, a strictly archival review paper by Barachevsky ${ }^{6}$ from Russia deals quite practically with electrooptic polymers and photopolymerizable systems among other materials of photoinduced birefringence. This paper can also be found in SPIE/PL Proceedings, Volume 2208.

Consequently, we recommend reading this special section in combination with the conference/proceedings papers cited previously.

To construct our special section to be as representative as possible, we had many discussions with potential contributors. It was self-evident that they were more constructive in the editors' country than abroad, and thus the proportion between the Polish authors and contributors from other countries is as high as 8 to 3 . The former, however, are selected from different sources: the Institute of Physical Chemistry of the Polish Academy of Sciences (PAS), Warsaw; the Institute of Fundamental Technological Research of PAS, Warsaw; Centre of Molecular and Macromolecular Studies of PAS, Lódź; Adam Mickiewicz University, Poznań; Poznań University of Technology; Warsaw University of Technology; and Military University of Technology, Warsaw.

Among the 11 papers, the first is theoretical and deals with some basic optical (absorption) properties of simple polymeric structures, referred to as cumulenes. These play a practical role in polymer paints. The second paper is also theoretical and includes some methodical aspects and practical references concerning the use of Mueller's matrix calculus in the polarized-light modulation spectroscopy of polymers. The next six papers report a number of experimental studies 
and measurements of structural, chemical, physical, and optical properties of various polymers by using polarized-light microscopy, optical Kerr effect, light scattering methods, optical spectroscopy, and waveguide techniques. The last three papers qualify as engineering contributions. One of them deals with photoelastic polymers and their mechanical and optical properties suitable for experiments in photomechanics. The next one presents an optical force sensor based on a polymer disk, and the last paper discusses optical and electro-optical properties of polymer-dispersed liquid crystals (PDLCs).

This scope shows that the special section covers the topic area as widely as possible within a limited space. First, anisotropic or birefringent properties of polymers suitable for different practical applications are discussed, then nonlinear optics using polymer materials is taken into consideration, and examples of modern optopolymeric devices are described. Here it is important to note that nonlinear optical polymers produce a great interest in integrated optics. For instance, an integrated optical modulator or switch can be made of some polymeric layers, the properties of which are electrically modified, and thus they operate as electro-optically active elements. Their effective design depends on the precise knowledge of the refractive indices of the layers, including their nonlinear terms. The paper by Vitrant et al. approaches this modern technology (see also Ref. 2).

A large area in the field of this special section is physics or optics of both textile and other (optical, composite) polymer fibers. One of us (Pluta) was strongly interested in preparing an archival paper on variable-wavelength double-refracting microinterferometry and optical Fourier transform microscopy of highly birefringent polymer fibers, but this idea did not ensue. Instead, a number of earlier papers on that subject ${ }^{7-10}$ are included with the references for the readers interested in this matter.

We are grateful to the authors and reviewers who were highly cooperative with this special section. We also transfer many thanks to Brian J. Thompson and his Optical Engineering staff for their patience and help with our editorial process and for publishing this composition of papers on optics in polymer science and technology.

\section{References}

1. G. Vitrant, R. Reinisch, and F. Kajzar, "M-line spectroscopy for nonlinear waveguide characterization," in Refractometry, M. Pluta and M. Szyjer, Eds., Proc. SPIE 2208, 116-118 (1995).

2. E. Lörincz and R. Klug, Refractometry of weakly absorbing multilayers via reflectometry," in Refractometry, M. Pluta and M. Szyjer, Eds., Proc. SPIE 2208, 162-171 (1995).

3. F. Carreño and E. Barnabeu, "Statistical algorithm to obtain refractive index and thickness from spectrophotometric interference patterns," in Refractometry, M. Pluta and M Szyjer, Eds., Proc. SPIE 2208, 77-87 (1995).

4. P. Buyanovskaya, "Measurement of refraction and absorption indices of thin polymer film on the transparent semiconductor substrate via FTIR spectroscopy," in Refractometry, M. Pluta and M Szyjer, Eds., Proc. SPIE 2208, 172-176 (1995).
5. I. I. Gancheryonok and A. V. Lavrinenko, "Reflection variant of nonlinear polarization spectroscopy," Opt. Appl. 25(2), in press (1995).

6. V.A. Barachevsky, "Photoinduced birefringence and its application," in Refractometry, M. Pluta and M Szyjer, Eds., Proc. SPIE 2208, 182-193 (1995).

7. M. Pluta, "Variable wavelength microinterferometry of textile fibres," J. Microscopy 149(2), 97-115 (1987).

8. M. Pluta, "Birefringence of aramid fibres," Polym. Commun. 33(7), 1553-1555 (1992).

9. M. Pluta, "Quasi-object-adapted variable-wavelength doublerefracting microscope interferometry," Opt. Eng. 31(3), 402-421 (1992).

10. M. Pluta, "Optical Fourier transforms of birefringent fibres. I. Polymeric textile fibres," J. Modern Opt. 34(11), 1451-1470 (1987).

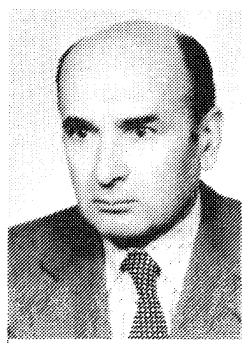

Maksymilian Pluta received his higher education in physics from the Jagiellonian University, Krakow, and Warsaw University, Poland, from 1949 to 1954. In 1954 he joined the Central Laboratory of Optics (now the Institute of Applied Optics), where he has been head and organizer of the Department of Physical Optics. He earned the $\mathrm{PhD}$ degree and Habilitation in applied optics and received, in 1982, the scientific title and position of Professor.

For many years he has been associated with the Institute of Physics at the Warsaw University of Technology, where he is active as a professor conferring doctoral degrees in applied optics. $\mathrm{He}$ is the author of several scientific books, among which the threevolume monograph on advanced light microscopy is predominant; many tens of journal and conference papers; and 26 patents. He has been a member and fellow of SPIE since 1985 and 1992, respectively, and currently is president of the SPIE Poland chapter. His various recent interests include optical studies of polymers, especially double-refracting microinterferometry and polarizedlight microscopy of polymer fibers, which are his specialty.

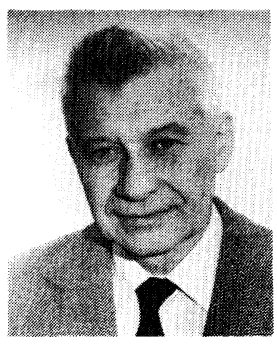

Andrzej Wasiak is a senior research fellow at the Institute of Fundamental Technological Research, Polish Academy of Sciences, Warsaw. He received his MS degree in 1961 from the University of Warsaw, Department of Chemistry, and his $\mathrm{PhD}$ in 1974 in technical sciences (polymer science) from the Polish Academy of Sciences. He has been with the Department of Crystallography, the University of Warsaw; the Research Institute of General Chemistry in Warsaw; and the Laboratory of Polymer Physics at the Institute of Fundamental Technological Research of the Polish Academy of Sciences. He has been a postdoctoral associate at the University of Massachusetts and a visiting scientist at the Department of Polymer Chemistry, Kyoto University, Japan, and at the Department of Applied Physics, University of Duisberg, Federal Republic of Germany. Wasiak's interests are in experimental studies of structure formation in polymers by means of x-ray diffraction, scattering of visible light, quantitative aspects of microscopy, and studies of anisotropy of polymeric materials. 